\title{
Introduction and purpose of the tutorial series Python for Research in Psychology
}

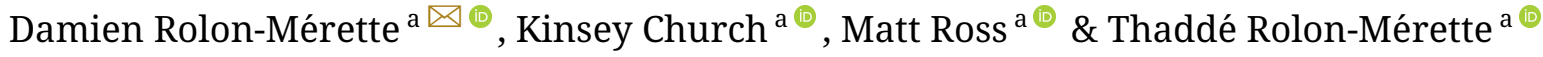 \\ ${ }^{\mathrm{a}}$ University of Ottawa
}

\begin{abstract}
In the era of advanced computers and state-of-the-art software, there have been great strides in the domains of research and engineering. These advancements have been made possible through the popularity, accessibility, and integration of computer programming. This has made automating tasks, analysing results through complex statistical analysis, and the development of more advanced artificial intelligence possible. However, in psychology, computer programming is still being underutilized. A major factor contributing to this is the steep learning curve for programming that is compounded by a lack of tutorials and knowledge specifically geared towards psychology. Thus, the purpose of this series is to bridge this gap and lower the learning barrier for researchers. This series invites anyone to send their own tutorials and contribute towards building a comprehensive guide to programming for psychological research in one of the most versatile languages: Python! For submission details, please see the guidelines below.
\end{abstract}

Keywords $\backsim$ Python, Psychology. Tools $\backsim$ Python.

Editorial.

drolo083@uottawa.ca

10.20982/tqmp.16.5.S001

\section{Purpose of the Python for Research in Psychology se- ries}

The word "research" is often accompanied by the concept of experiments, which require an individual to create and implement different research designs to capture the essence of the world through small instances. As any scientist may testify, this process can be at times daunting and mundane. In the field of psychology, research may involve repeating stimuli presentations manually to participants, manually recording and analyzing interviews, and creating and scoring questionnaires. This is the way these research methods have been conducted over the past decades, but these tiresome tasks could be expedited through automation and computer science.

Currently, most software is expensive and only offers a limited approach when it comes to capturing and analyzing data. With its versatility, open-source nature, and widespread community, Python has become one of the most popular programming languages to learn. Python can be used to build, collect, and automate projects of all sizes.
This coding language allows users to build elaborate research designs, automate data collection through machine learning, and perform advanced statistical analysis with ease. By learning Python, you are not only learning a valuable skill, but it can also lead to increased research productivity.

So why is programming in psychology not more widespread? Unfortunately, most researchers are unsure of where and how to get started. They may not see the potential applications for their own research or how to get help if things go awry. They are intimidated by the learning curve and do not know where to get concrete examples and practice material related to psychology. These doubts are often hard to overcome because there are so many resources related to Python for engineering and other fields. Finding the right materials for psychological research can be overwhelming. This special Python tutorial series has been created to remedy this, to facilitate the learning curve, and to regroup relevant concepts related to psychology. With a range from novice introductory tutorials to more advanced topics, such as implementations 
of machine learning algorithms for data analysis, this series will provide an ideal space for any researcher to learn and perfect their Python programming skills. These tutorials will save researchers valuable time by giving them the tools to automate daunting tasks as well as create more sophisticated research designs and analyses. Following Python's open-source qualities, this series invites anyone to submit relevant and important concepts related to psychology and Python.

\section{Submitting to this tutorial series}

If you are interested in submitting a tutorial to be considered for this series, please ensure it meets the following criteria:

- The tutorial is related to research in Psychology and in Python

- Each submission must follow the APA format (see Myers et al., 2019, for additional guidelines)

- Each tutorial must also be transmitted in $\mathrm{LT}_{\mathrm{E}} \mathrm{X}$ format

- All submissions must be in English

- The maximum submission length is 20 pages

- If a tutorial on a similar topic has already been published in TQMP, motivate in your cover letter why a second paper should be considered by the editorial team

\section{Authors' note}

The authors can be contacted by email at (Damien Rolon-Mérette) drolo083@uottawa.ca, (Kinsey Church) kchur026@uottawa.ca, (Matt Ross) mross094@uottawa.ca, or (Thaddé Rolon-Mérette) trolo068@uottawa.ca.

\section{References}

Myers, M., Paiz, J., Angeli, E., Wagner, J., Lawrick, E., Moore, K., ... Keck, R. (2019). APA style workshop // Purdue Writing Lab. Retrieved December 12, 2019, from https://owl.purdue. edu/owl/research_and_ citation/apa_style/apa_formatting_and_style_guide/ general_format.html

\section{Citation}

Rolon-Mérette, D., Ross, M., Rolon-Mérette, T., \& Church, K. (2020). Introduction and purpose of the tutorial series python for research in psychology. The Quantitative Methods for Psychology, 16(5), S1-S3. doi:10.20982/tqmp.16.5.S001

Copyright @ 2020, Rolon-Mérette, Ross, Rolon-Mérette, and Church. This is an open-access article distributed under the terms of the Creative Commons Attribution License (CC BY). The use, distribution or reproduction in other forums is permitted, provided the original author(s) or licensor are credited and that the original publication in this journal is cited, in accordance with accepted academic practice. No use, distribution or reproduction is permitted which does not comply with these terms.

Received: 11/08/2020 